\title{
Polaritons in a nonideal array of ultracold quantum dots
}

\author{
V.V. Rumyantsev ${ }^{1,2}$, S.A. Fedorov ${ }^{1}$, and K.V. Gumennyk ${ }^{1}$ \\ ${ }^{l}$ O.O. Galkin Donetsk Institute for Physics and Engineering NAS of Ukraine, Kyiv 03680, Ukraine \\ ${ }^{2}$ Mediterranean Institute of Fundamental Physics, 00047 Marino, Rome, Italy \\ E-mail: kgumennyk@gmail.com
}

Received December 14, 2015, published online March 23, 2016

\begin{abstract}
We develop a numerical model for a defect-containing square lattice of microcavities with embedded ultracold atomic clusters (quantum dots). It is assumed that certain fractions of quantum dots and cavities are absent, which leads to transformation of polariton spectrum of the overall structure. The dispersion relations for polaritonic modes are derived as functions of defect concentrations and on this basis the band gap, the effective masses of lower and upper dispersion branch polaritons as well as their densities of states are evaluated.
\end{abstract}

PACS: $42.60 . \mathrm{Da}$ Resonators, cavities, amplifiers, arrays, and rings;

42.70.Qs Photonic bandgap materials;

42.79.Gn Optical waveguides and couplers;

78.67.Hc Quantum dots.

Keywords: microcavities, quantum dots, polaritons, band gap, effective mass, density of state.

\section{Introduction}

Designing and utilization of novel materials for manufacturing of the sources of coherent irradiation is currently a vast interdisciplinary area, which spans various theoretical and fundamental aspects of laser physics, condensed matter physics, nanotechnology, chemistry as well information science [1,2]. Physical realization of corresponding devices requires the ability to manipulate the group velocity of propagation of electromagnetic pulses, which is accomplished by the use of the so-called polaritonic crystals $[3,4]$. The latter represent a particular type of photonic crystals [5] featured by a strong coupling between quantum excitations in the medium (excitons) and optical fields.

An example of polaritonic structure can be given by a spatially periodic system of coupled microcavities [6]. An interest for optical modes in microcavity arrays has been growing lately due to the enhancement of optoelectronic devices $[7,8]$. In this connection the defect-based resonators in photonic crystals deserve special attention [9]. In [10] it was shown that such resonators can form a strong coupling with quantum dots. Refs. 3,4 gave a theoretical analysis of the formation of quantum solitons coupled to lower dispersion branch (LDB) polaritons in a chain of microcavities. The authors suggest that such systems can be particularly appealing for the purposes of quantum information processing. Microcavity systems can also be employed for the construction of highly accurate optical clockworks [11-13].

It is worth stressing that the conventional polaritonic model $[3,4]$ of the atomic-optical interaction is only applicable to the case of ultracold atoms with frozen-out degrees of freedom. The corresponding approximation is valid when the number of atoms contained in individual cavities is relatively small $\left(N \leq 10^{4}\right)$ [14]. Parameter $g$ of the strong atomic-optical interaction must satisfy the condition

$$
g \gg 2 \pi / \tau_{\mathrm{coh}},
$$

i.e., in each cavity $g$ should much exceed the inverse coherence time $\tau_{\mathrm{coh}}$ of the atomic-optical system [15]. Physically, $\tau_{\mathrm{coh}}$ is the time of thermodynamic equilibration of the atomic system, which interacts with a quantized field in a polaritonic crystal. The said inequality holds at temperatures of the order of several $\mathrm{mK}$, when the spectral line broadening is negligible and so there are pure (thermodynamically equilibrium) quantum states of the atomic-field system.

The currently rapidly evolving field of endeavor is the photonics of imperfect structures. Some of our recent works are devoted to optical activity of imperfect photonic crystals [16] as well as to dispersion of exciton-like electromagnetic excitations in nonideal (defect-containing) arrays of coupled microcavities $[17,18]$. Introduction of 
defects provides an additional powerful tool for controlling the propagation of electromagnetic excitations through photonic structures.

In what follows we use the previously developed concepts of photonic structures $[17,18]$ to investigate a nonideal polaritonic crystal constituted by a topologically ordered assembly of coupled microcavities with embedded quantum dots. It is assumed that certain portions of quantum dots and cavities are missing, which is viewed as the presence of defects. The polariton spectrum of the structure and the related quantities of interest (the band gap, the effective masses of polaritons as well as their densities of states) are investigated as functions of defect concentrations.

\section{Numerical model of a microcavity array}

A frequently used method of fabrication of polaritonic crystals is the trapping of two-level atoms in an ideal coupled-resonator optical waveguide (CROW) [3] or in a nonideal array of microcavities [18]. To keep our discussion sufficiently general let us first consider a two-dimensional lattice of microcavities with an arbitrary number $\sigma$ of sublattices and then tackle the important particular case of $\sigma=1$. Assume that the $\alpha$ th sublattice is composed of $s(\alpha)$ types of randomly distributed resonators, each of whom contains a quantum dot (a one-level atomic cluster) pertaining to one of $r(\alpha)$ types. Quantum dots interact with resonator-localized quantized electromagnetic fields and each of tunnel-coupled resonators possesses a single optical mode. In the coordinate representation the Hamiltonian of the described superstructure writes:

$$
\hat{H}=\hat{H}_{\mathrm{at}}+\hat{H}_{\mathrm{ph}}+\hat{H}_{\mathrm{int}} .
$$

In (1) Hamiltonians of the atomic (quantum dot) subsystem $\hat{H}_{\text {at }}$, photonic (microcavity) subsystem $\hat{H}_{\mathrm{ph}}$ and their interaction $\hat{H}_{\text {int }}$ equal correspondingly to $\hat{H}_{\mathrm{at}}=\sum_{n} \hat{H}_{\mathrm{at}, n}+$ $+\frac{1}{2} \sum_{n, m} \hat{V}_{n m}, \quad \hat{H}_{\mathrm{ph}}=\sum_{n} \hat{H}_{\mathrm{ph}, n}-\frac{1}{2} \sum_{n, m} \hat{A}_{n m}, \quad \hat{H}_{\mathrm{int}}=\sum_{n} \hat{G}_{n}$, where $\hat{H}_{\mathrm{at}, n}$ is the Hamiltonian of a stationary (ultracold) quantum dot embedded at the $n$th cavity, $\hat{V}_{n m}$ is the operator of Coulomb interaction between quantum dots at the $n$th and $m$ th cavities, $\hat{H}_{\mathrm{ph}, n}$ defines the state of electromagnetic excitation localized at the $n$th cavity, $\hat{A}_{n m}$ describes an overlap of optical fields of the $n$th and $m$ th cavities (and hence the transfer probability of the corresponding electromagnetic excitation). Writing interaction operator $\hat{H}_{\text {int }}$ as a sum of unitary operators $\hat{G}_{n}$ is justified under the assumption that each of cavity-localized electromagnetic excitations interacts only with the quantum dot embedded at the same cavity. Complex indices $n$ and $m$ are defined by expressions $n \equiv(\mathbf{n}, \boldsymbol{\alpha}), \quad m \equiv(\mathbf{m}, \boldsymbol{\beta})$, where two-dimensional vectors $\mathbf{n}$ and $\mathbf{m}$ define positions of elementary cells in the superlattice, whereas $\alpha$ and $\beta$ numerate sublattices and assume values $1,2,3, \ldots \sigma$.

Let us calculate in accordance with $[19,20]$ the energy spectrum of the studied system by writing down its Hamiltonian $\hat{H}$ as a sum of the following second quantization operators:

$$
\begin{aligned}
& \hat{H}_{\mathrm{at}}=\sum_{n, f} \varepsilon_{n f}^{\mathrm{at}} \hat{b}_{n f}^{+} \hat{b}_{n f}+\frac{1}{2} \sum_{n \neq m} \sum_{f, g} \sum_{h, l} \hat{b}_{n f}^{+} \hat{b}_{m g}^{+}\left\langle\varphi_{n f}^{\mathrm{at}} \varphi_{m g}^{\mathrm{at}}\left|V_{n m}\right| \varphi_{m h}^{\mathrm{at}} \varphi_{n l}^{\mathrm{at}}\right\rangle \hat{b}_{m h} \hat{b}_{n l}, \\
& \hat{H}_{\mathrm{ph}}=\sum_{n, \mu} \varepsilon_{n \mu}^{\mathrm{ph}} \hat{\phi}_{n \mu}^{+} \hat{\phi}_{n \mu}-\frac{1}{2} \sum_{n \neq m} \sum_{\mu, v \lambda, \rho} \hat{\phi}_{n \mu}^{+} \hat{\phi}_{m v}^{+}\left\langle\varphi_{n \mu}^{\mathrm{ph}} \varphi_{m v}^{\mathrm{ph}}\left|A_{n m}\right| \varphi_{m \lambda}^{\mathrm{ph}} \varphi_{n \rho}^{\mathrm{ph}}\right\rangle \hat{\phi}_{m \lambda} \hat{\phi}_{n \rho}, \\
& \hat{H}_{\mathrm{int}}=\sum_{n, f, g \mu, v} \sum_{n g}^{+} \hat{b}_{n \mu}^{+}\left\langle\varphi_{n g}^{\mathrm{at}} \varphi_{n \mu}^{\mathrm{ph}}\left|\hat{G}_{n}\right| \varphi_{n f}^{\mathrm{at}} \varphi_{n v}^{\mathrm{ph}}\right\rangle \hat{b}_{n f} \hat{\phi}_{n v} .
\end{aligned}
$$

Here $\varepsilon_{n f}^{\text {at }}, \varepsilon_{n \mu}^{\mathrm{ph}}$ are the eigenvalues of operators $\hat{H}_{\mathrm{at}}$ and $\hat{H}_{\text {ph }}$, respectivety. Wave functions $\varphi_{n f}^{\text {at }}, \varphi_{n \lambda}^{\text {ph }}$ characterize the states of quantum dot and electromagnetic field at the $n t h$ resonator, while the Hermitian conjugate (nonHermitian) creation and annihilation operators $\hat{b}_{n f}^{+}, \hat{b}_{n f}$, $\hat{\phi}_{n \mu}^{+}, \hat{\phi}_{n \mu}$ describe, correspondingly, the states $f$ of quantum dot and the states $\mu$ of electromagnetic field at the $n$th cavity. $\hat{b}_{n f}^{+}, \hat{b}_{n f}$ and $\hat{\phi}_{n \mu}^{+}, \hat{\phi}_{n \mu}$ are Pauli operators which satisfy the following commutation relations

$$
\hat{b}_{n f} \hat{b}_{m g}^{+}+\hat{b}_{m g}^{+} \hat{b}_{n f}=1, \quad \hat{b}_{n f} \hat{b}_{m g}=\hat{b}_{n f}^{+} \hat{b}_{m g}^{+}=0
$$

for $n f=m g$ and

$$
\hat{b}_{n f} \hat{b}_{m g}-\hat{b}_{m g} \hat{b}_{n f}=\hat{b}_{n f} \hat{b}_{m g}^{+}-\hat{b}_{m g}^{+} \hat{b}_{n f}=0
$$

for $n f \neq m g$ (analogous commutation relations hold for $\hat{\phi}_{n \mu}^{+}$and $\left.\hat{\phi}_{n \mu}\right)$.

Let us make a reasonable assumption that the densities of excited states of elements in both constituent subsystems (atomic and resonator) is a small quantity. This allows to simplify the energy operator (1)-(2) by approximating Pauli operators with Bose operators

$$
\begin{array}{cl}
\hat{b}_{n f}^{+} \hat{b}_{n 0} \approx \hat{B}_{n f}^{+}, & \hat{b}_{n 0}^{+} \hat{b}_{n f} \approx \hat{B}_{n f}, \\
\hat{\phi}_{n \mu}^{+} \hat{\phi}_{n 0} \approx \hat{\Psi}_{n \mu}^{+}, & \hat{\phi}_{n 0}^{+} \hat{\phi}_{n \mu} \approx \hat{\Psi}_{n \mu} .
\end{array}
$$


Next, since we are constructing a one-level model, indices $f, g, h, l$ in (2) should assume the values 0 and $a$, while indices $\mu, v, \lambda, \rho$ assume values 0 and 1 . Therefore within the Heitler-London approximation the quadratic (in
$\hat{B}_{n f}$ and $\hat{\Psi}_{n \mu}$ ) part of Hamiltonian (2) is given by the following expressions:

$$
\begin{aligned}
& \hat{H}_{\mathrm{at}}^{H-L}=\sum_{n}\left(\Delta \varepsilon_{n a}+\sum_{m}\left(\left\langle\varphi_{n a}^{\mathrm{at}} \varphi_{m 0}^{\mathrm{at}}\left|\hat{V}_{n m}\right| \varphi_{m 0}^{\mathrm{at}} \varphi_{n a}^{\mathrm{at}}\right\rangle-\left\langle\varphi_{n 0}^{\mathrm{at}} \varphi_{m 0}^{\mathrm{at}}\left|\hat{V}_{n m}\right| \varphi_{m 0}^{\mathrm{at}} \varphi_{n 0}^{\mathrm{at}}\right\rangle\right)\right) \hat{B}_{n a}^{+} \hat{B}_{n a}+ \\
& +\sum_{n, m}\left\langle\varphi_{n 0}^{\mathrm{at}} \varphi_{m a}^{\mathrm{at}}\left|\hat{V}_{n m}\right| \varphi_{m 0}^{\mathrm{at}} \varphi_{n a}^{\mathrm{at}}\right\rangle \hat{B}_{n a}^{+} \hat{B}_{m a} \equiv \sum_{n} \hbar \omega_{n a}^{\mathrm{at}} \hat{B}_{n a}^{+} \hat{B}_{n a}+\sum_{n, m} V_{n m}^{(a)} \hat{B}_{n a}^{+} \hat{B}_{m a}, \\
& \hat{H}_{\mathrm{ph}}^{H-L}=\sum_{n}\left(E_{n 1}-\sum_{m}\left(\left\langle\varphi_{n 1}^{\mathrm{ph}} \varphi_{m 0}^{\mathrm{ph}}\left|\hat{A}_{n m}\right| \varphi_{m 0}^{\mathrm{ph}} \varphi_{n 1}^{\mathrm{ph}}\right\rangle-\left\langle\varphi_{n 0}^{\mathrm{ph}} \varphi_{m 0}^{\mathrm{ph}}\left|\hat{A}_{n m}\right| \varphi_{m 0}^{\mathrm{ph}} \varphi_{n 0}^{\mathrm{ph}}\right\rangle\right)\right) \hat{\Psi}_{n 1}^{+} \hat{\Psi}_{n 1}- \\
& -\sum_{n, m}\left\langle\varphi_{n 0}^{\mathrm{ph}} \varphi_{m 1}^{\mathrm{ph}}\left|\hat{A}_{n m}\right| \varphi_{m 0}^{\mathrm{ph}} \varphi_{n 1}^{\mathrm{ph}}\right\rangle \hat{\Psi}_{n 1}^{+} \hat{\Psi}_{m 1} \equiv \sum_{n} \hbar \omega_{n 1}^{\mathrm{ph}} \hat{\Psi}_{n 1}^{+} \hat{\Psi}_{n 1}-\sum_{n, m} A_{n m} \hat{\Psi}_{n 1}^{+} \hat{\Psi}_{m 1}, \\
\hat{H}_{\mathrm{int}}^{H-L}= & \sum_{n} \hat{\Psi}_{n 1}^{+} \hat{B}_{n a}\left\langle\varphi_{n 0}^{\mathrm{at}} \varphi_{n 1}^{\mathrm{ph}}\left|\hat{G}_{n}\right| \varphi_{n a}^{\mathrm{at}} \varphi_{n 0}^{\mathrm{ph}}\right\rangle+\hat{\Psi}_{n 1} \hat{B}_{n a}^{+}\left\langle\varphi_{n a}^{\mathrm{at}} \varphi_{n 0}^{\mathrm{ph}}\left|\hat{G}_{n}\right| \varphi_{n 0}^{\mathrm{at}} \varphi_{n 1}^{\mathrm{ph}}\right\rangle \equiv \sum_{n} g_{n}\left(\hat{\Psi}_{n 1}^{+} \hat{B}_{n a}+\hat{\Psi}_{n 1} \hat{B}_{n a}^{+}\right) .
\end{aligned}
$$

In (3) and (4) appear the frequency characteristics $\omega_{n 1}^{\mathrm{ph}}$, $\omega_{\mathbf{n} \alpha}^{\mathrm{at}}$ of resonator and atomic subsystems as well as the matrix of resonant coupling:

$$
\begin{aligned}
\left\langle\varphi_{n 0}^{\mathrm{at}} \varphi_{m a}^{\mathrm{at}}\left|\hat{V}_{n m}\right| \varphi_{m 0}^{\mathrm{at}} \varphi_{n a}^{\mathrm{at}}\right\rangle & \equiv V_{n m}^{(a)}, \\
\left\langle\varphi_{n 0}^{\mathrm{ph}} \varphi_{m 1}^{\mathrm{ph}}\left|\hat{A}_{n m}\right| \varphi_{m 0}^{\mathrm{ph}} \varphi_{n 1}^{\mathrm{ph}}\right\rangle & \equiv A_{n m} .
\end{aligned}
$$

In (5) it is taken into account that the wave functions of quantum dots and electromagnetic fields are real-valued and hence

$$
\left\langle\varphi_{n 0}^{\mathrm{at}} \varphi_{n 1}^{\mathrm{ph}}\left|\hat{G}_{n}\right| \varphi_{n a}^{\mathrm{at}} \varphi_{n 0}^{\mathrm{ph}}\right\rangle=\left\langle\varphi_{n a}^{\mathrm{at}} \varphi_{n 0}^{\mathrm{ph}}\left|\hat{G}_{n}\right| \varphi_{n 0}^{\mathrm{at}} \varphi_{n 1}^{\mathrm{ph}}\right\rangle \equiv g_{n} .
$$

The deviation from ideality of the considered system consists in disordering of its resonant and atomic subsystems. Mathematically this is reflected in the fact that the quantities $\omega_{n 1}^{\mathrm{ph}}, \omega_{n a}^{\mathrm{at}}, V_{n m}^{(a)}, A_{n m}$ and $g_{n}$ are configurationally dependent and therefore Hamiltonian (1) is not translation invariant. One of the methods of obtaining the spectra of quasiparticle excitations in disordered systems with randomly distributed elements consists in finding the poles of configurationally averaged resolvent of the appro- priate Hamiltonian [21]. The said resolvent is translation invariant and so the corresponding elementary excitation spectrum can be described by a wave vector $\mathbf{k}$. To carry out the necessary calculation one should inevitably adopt a certain approximation, whose choice is dictated by the specifics of the studied system. A widespread tool for evaluation of quasiparticle states in disordered media is the virtual crystal approximation (VCA) [21,22]. It is particularly suitable for tracing the effect of defect concentrations on the specifics of the spectrum and the related quantities. Within the VCA the averaged resolvent equals to the resolvent of the averaged Hamiltonian, and it is the latter, which needs to be diagonalized in order to calculate the spectrum. In what follows we use this approximation to analyze electromagnetic excitations and optical characteristics of the studied microcavity superstructure.

Let us, by analogy with $[17,18]$ express the configurationally dependent quantities $\omega_{n a}^{\mathrm{at}} \equiv \omega_{\mathbf{n} \alpha}^{\mathrm{at}}, \quad \omega_{n 1}^{\mathrm{ph}} \equiv \omega_{\mathbf{n} \alpha}^{\mathrm{ph}}$, $V_{n m}^{(a)}, A_{n m}$ and $g_{n}$ through the random quantities $\eta_{\text {at,no }}^{v(\alpha)}$ $\left(\eta_{\mathrm{ph}, \mathbf{n} \alpha}^{v(\alpha)}\right)$ :

$$
\begin{array}{rlrl}
\omega_{\mathbf{n} \alpha}^{\mathrm{at}} & =\sum_{v(\alpha)=1}^{r(\alpha)} \omega_{\mathrm{at}, \alpha}^{v(\alpha)} \eta_{a t, \mathbf{n} \alpha}^{v(\alpha)} ; & V_{\mathbf{n} \alpha \mathbf{m} \beta}^{(a)}=\sum_{v(\alpha), \mu(\beta)=1}^{r(\alpha) r(\beta)} V_{\alpha \beta}^{v(\alpha) \mu(\beta)}(\mathbf{n}-\mathbf{m}) \eta_{\mathrm{at}, \mathbf{n} \alpha}^{v(\alpha)} \eta_{\mathrm{at}, \mathbf{m} \beta}^{\mu(\beta)}, \\
\omega_{\mathbf{n} \alpha}^{\mathrm{ph}}=\sum_{v(\alpha)=1}^{s(\alpha)} \omega_{\mathrm{ph}, \alpha}^{v(\alpha)} \eta_{\mathrm{ph}, \mathbf{n} \alpha}^{v(\alpha)} ; & A_{\mathbf{n} \alpha \mathbf{m} \beta}=\sum_{v(\alpha), \mu(\beta)=1}^{s(\alpha) s(\beta)} A_{\alpha \beta}^{v(\alpha) \mu(\beta)}(\mathbf{n}-\mathbf{m}) \eta_{\mathrm{ph}, \mathbf{n} \alpha}^{v(\alpha)} \eta_{\mathrm{ph}, \mathbf{m} \beta}^{\mu(\beta)} .
\end{array}
$$

Configurational dependence of both constituent subsystems is reflected in the quantity $g_{n}$

$$
g_{n}=\sum_{v(\alpha)=1 \mu(\alpha)=1}^{r(\alpha)} \sum_{\alpha}^{s(\alpha)} g_{\alpha}^{v(\alpha) \mu(\alpha)} \eta_{\mathrm{at}, \mathbf{n} \alpha}^{v(\alpha)} \eta_{\mathrm{ph}, \mathbf{n} \alpha}^{\mu(\alpha)}
$$


where $\eta_{\mathrm{at}, \mathbf{n} \alpha}^{v(\alpha)}=1 \quad\left(\eta_{\mathrm{ph}, \mathbf{n} \alpha}^{v(\alpha)}=1\right)$ if the $n \alpha$-th site is occupied by a quantum dot (resonator) of the $v(\alpha)$-th type and $\eta_{\mathrm{at}, \mathbf{n} \alpha}^{v(\alpha)}=0 \quad\left(\eta_{\mathrm{ph}, \mathbf{n} \alpha}^{v(\alpha)}=0\right)$ in all other cases. On the assumption that the ordering states of the two subsystems are in- dependent of each other we arrive at the following expressions for the configurationally averaged quantities

$$
\begin{aligned}
& \left\langle\omega_{\mathbf{n} \alpha}^{\mathrm{at}}\right\rangle=\sum_{v(\alpha)=1}^{r(\alpha)} \omega_{\mathrm{at}, \alpha}^{v(\alpha)} C_{\mathrm{at}, \alpha}^{v(\alpha)} ;\left\langle V_{\mathbf{n} \alpha \mathbf{m} \beta}^{(a)}\right\rangle=\sum_{v(\alpha), \mu(\beta)=1}^{r(\alpha) r(\beta)} V_{\alpha \beta}^{v(\alpha) \mu(\beta)}(\mathbf{n}-\mathbf{m}) C_{\mathrm{at}, \alpha}^{v(\alpha)} C_{\mathrm{at}, \beta}^{\mu(\beta)}, \\
& \left\langle\omega_{\mathbf{n} \alpha}^{\mathrm{ph}}\right\rangle=\sum_{v(\alpha)=1}^{s(\alpha)} \omega_{\mathrm{ph}, \alpha}^{v(\alpha)} C_{\mathrm{ph}, \alpha}^{v(\alpha)} ;\left\langle A_{\mathbf{n} \alpha \mathbf{m} \beta}\right\rangle=\sum_{v(\alpha), \mu(\beta)=1}^{s(\alpha) s(\beta)} A_{\alpha \beta}^{v(\alpha) \mu(\beta)}(\mathbf{n}-\mathbf{m}) C_{\mathrm{ph}, \alpha}^{v(\alpha)} C_{\mathrm{ph}, \beta}^{\mu(\beta)}, \\
& \left\langle g_{n}\right\rangle=\sum_{v(\alpha)=1 \mu(\alpha)=1}^{r(\alpha)} \sum_{\alpha}^{s(\alpha)} g_{\alpha}^{v(\alpha) \mu(\alpha)} C_{\mathrm{at}, \alpha}^{v(\alpha)} C_{\mathrm{ph}, \alpha}^{\mu(\alpha)},
\end{aligned}
$$

where angular brackets denote the averaging procedure. $C_{\mathrm{at}, \alpha}^{\mathrm{v}(\alpha)}\left(C_{\mathrm{ph}, \alpha}^{\mathrm{v}(\alpha)}\right)$ and $C_{\mathrm{at}, \beta}^{\mu(\beta)}\left(C_{\mathrm{ph}, \beta}^{\mu \beta}\right)$ denote concentrations of the $v(\alpha)$-th and $\mu(\beta)$-th type of elements of the atomic or resonator subsystems. There hold the obvious relations $\sum_{v(\alpha)=1}^{r(\alpha)} C_{\mathrm{at}, \alpha}^{\mathrm{v}(\alpha)}=1, \quad \sum_{v(\alpha)=1}^{s(\alpha)} C_{\mathrm{ph}, \alpha}^{v(\alpha)}=1$

Configurational averaging allows to "restore" the translation invariance of a nonideal superstructure (which is essentially the central idea of the VCA) and to characterize the eigenvalues and eigenfunctions of Hamiltonian $\langle H\rangle$ of the resulting virtual crystal by a wave vector $\mathbf{k}=\left(k_{x}, k_{y}, 0\right)$. In k-representation Hamiltonian $\langle H\rangle$ writes as

$$
\langle\hat{H}\rangle_{\mathbf{k}}=\left\langle\hat{H}_{\mathrm{at}}\right\rangle_{\mathbf{k}}+\left\langle\hat{H}_{\mathrm{ph}}\right\rangle_{\mathbf{k}}+\left\langle\hat{H}_{\mathrm{int}}\right\rangle_{\mathbf{k}}
$$

where

$$
\begin{aligned}
& \left\langle\hat{H}_{\mathrm{at}}\right\rangle_{\mathbf{k}}=\sum_{\alpha, \beta}\left[\hbar\left\langle\omega_{\mathbf{n} \alpha}^{\mathrm{at}}\right\rangle \delta_{\alpha \beta}+V_{\alpha \beta}^{(a)}(\mathbf{k})\right] \hat{B}_{\alpha a}^{+}(\mathbf{k}) \hat{B}_{\beta a}(\mathbf{k}), \\
& \left\langle\hat{H}_{\mathrm{ph}}\right\rangle_{\mathbf{k}}=\sum_{\alpha, \beta}\left[\hbar\left\langle\omega_{\mathbf{n} \alpha}^{\mathrm{ph}}\right\rangle \delta_{\alpha \beta}-A_{\alpha \beta}(\mathbf{k})\right] \hat{\Psi}_{\alpha 1}^{+}(\mathbf{k}) \hat{\Psi}_{\beta 1}(\mathbf{k}), \\
& \left\langle\hat{H}_{\mathrm{int}}\right\rangle_{\mathbf{k}}=\sum_{\alpha}\left\langle g_{\mathbf{n} \alpha}\right\rangle\left[\hat{\Psi}_{\alpha 1}^{+}(\mathbf{k}) \hat{B}_{\alpha a}(\mathbf{k})+\hat{\Psi}_{\alpha 1}(\mathbf{k}) \hat{B}_{\alpha a}^{+}(\mathbf{k})\right] .
\end{aligned}
$$

Here $V_{\alpha \beta}^{(a)}(\mathbf{k}), A_{\alpha \beta}(\mathbf{k}), \quad \hat{B}_{\alpha a}(\mathbf{k}), \quad \hat{\Psi}_{\alpha 1}(\mathbf{k})$ are the Fourier components of $\left\langle V_{\mathbf{n} \alpha \mathbf{m} \beta}^{(a)}\right\rangle,\left\langle A_{\mathbf{n} \alpha \mathbf{m} \beta}\right\rangle, \hat{B}_{n a}$ and $\hat{\Psi}_{n 1}$, respectively (cf. $[17,18])$.

Diagonalization of Hamiltonian $\langle H\rangle_{\mathbf{k}}$ by the use of Bogolyubov-Tyablikov transformation [19] yields the expressions for the energies of polariton excitations in the considered microcavity crystal with embedded quantum dots.

\section{Numerical results and discussion}

To make our further discussion more specific let us concentrate on polariton excitations in a defect-containing one-sublattice square Bravais lattice with period $d$ (Fig. 1).
The role of defects is played by vacancies contained in both atomic and cavity subsystems. In such a case $C_{\mathrm{at}, 1}^{(1)} \equiv C_{1}^{V}$ and $C_{\mathrm{ph}, 1}^{(1)} \equiv C_{2}^{V}$. Simplicity of the structure permits to reduce the somewhat cumbersome notations of the previous section to a more comprehensible form. Namely, we shall operate with the quantities $V_{11} \equiv V$, $A_{11} \equiv A, \quad g_{1}^{11} \equiv g, \quad \omega_{\mathrm{ph}, 1}^{1} \equiv \omega_{\mathrm{ph}}, \quad \omega_{\mathrm{at}, 1}^{1} \equiv \omega_{\mathrm{at}}$. The above

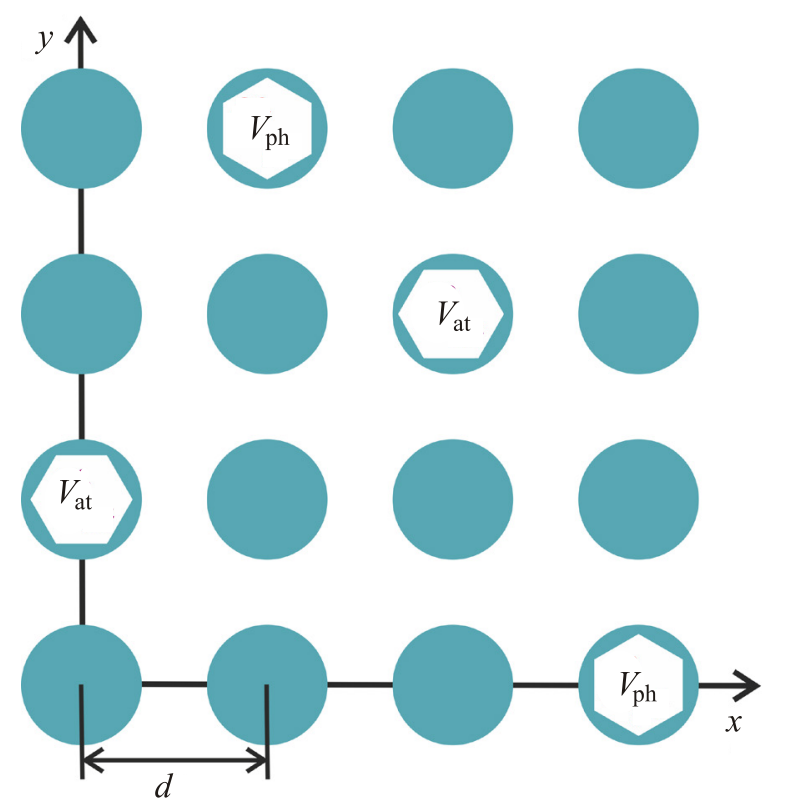

Fig. 1. Schematic of a model square one-sublattice array of microcavities. Solid circles denote defect-free cavities with embed-

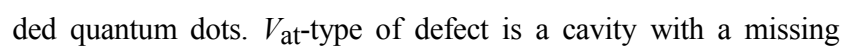

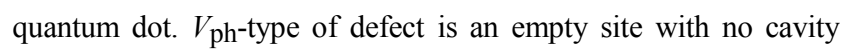
(and hence with no quantum dot, since atomic clusters can only reside at the existent cavities). Defect concentration in the atomic subsystem (i.e. concentration of sites with missing quantum dots) $C_{1}^{V}$ equals to the sum of concentrations of the $V_{\text {at- }}$ and $V_{\text {ph-types }}$ of defects. Defect concentration in the photonic subsystem (concentration of sites with missing cavities) $C_{2}^{V}$ equals to the $V_{\mathrm{ph} \text {-type }}$ defect concentration. There holds an obvious inequality $C_{1}^{V} \geq C_{2}^{V}$. 


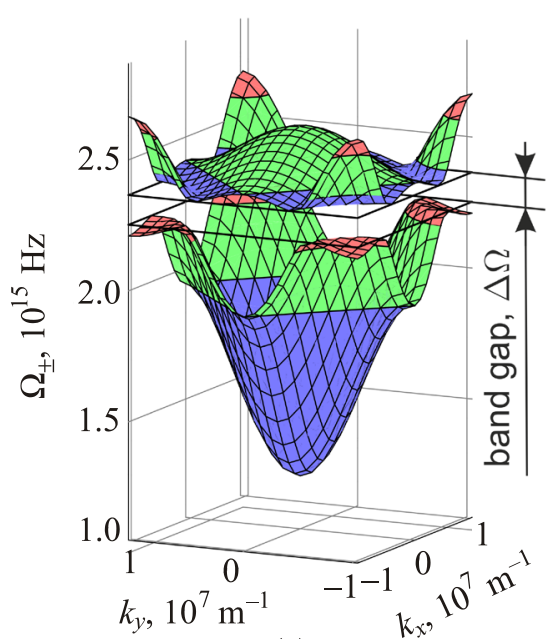

(a)

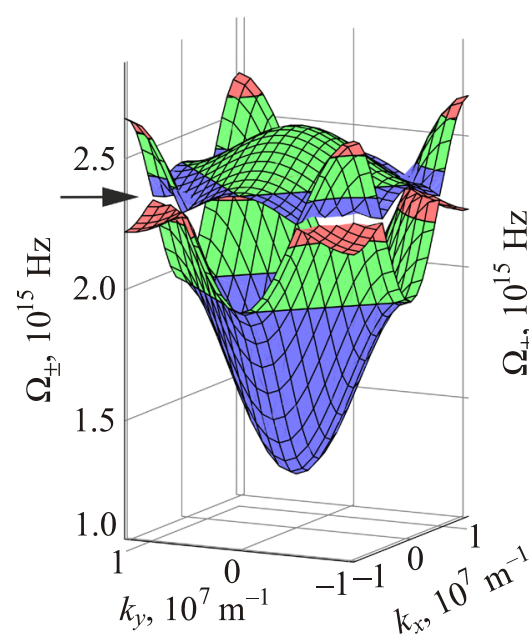

(b)

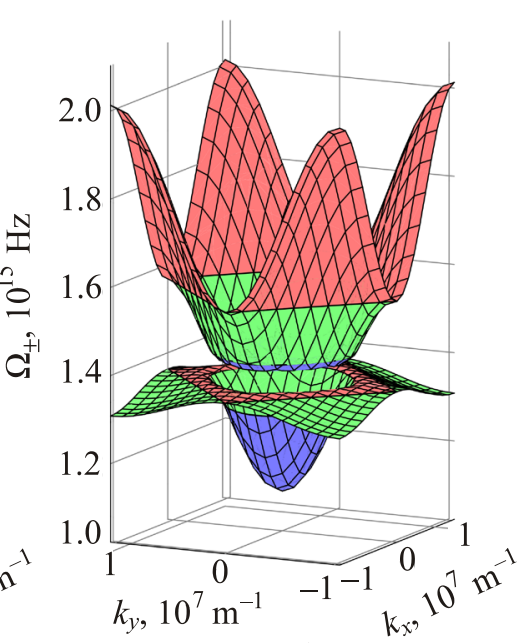

(c)

Fig. 2. (Color online) Dispersion bands of an ideal (a, b) microcavity array $\left(C_{1}^{V}=C_{2}^{V}=0\right.$ ) and a defect-containing (c) array $\left(C_{1}^{V}=0.43, C_{2}^{V}=0.2\right)$. (b) illustrates the narrowed "bottle neck" resulting from the decrease of parameter $g$ (responsible for interaction between the atomic and photonic subsystems) by a factor of 10 .

mentioned diagonalization procedure of Hamiltonian $\langle H\rangle_{\mathbf{k}}$ leads to a system of linear homogeneous equations, whose solvability condition is formulated as the equality of the following determinant to zero:

$$
\left\|\begin{array}{cc}
\hbar \omega^{\text {at }}(\mathbf{k})-\hbar \Omega(\mathbf{k}) & \left\langle g_{\mathbf{n}}\right\rangle \\
\left\langle g_{\mathbf{n}}\right\rangle & \hbar \omega^{\mathrm{ph}}(\mathbf{k})-\hbar \Omega(\mathbf{k})
\end{array}\right\|=0 .
$$

Here the energies of exciton exitations of the atomic (quantum dot) subsystem and of exciton-like electromagnetic exitations $[16,17]$ of the resonator (cavity) subsystem within the nearest-neighbor approximation equal correspondingly to:

$$
\begin{aligned}
& \hbar \omega^{\mathrm{at}}(\mathbf{k})=\hbar\left\langle\omega_{\mathbf{n}}^{\mathrm{at}}\right\rangle+2 V(d)\left(1-C_{1}^{V}\right)^{2}\left(\cos k_{x} d+\cos k_{y} d\right), \\
& \hbar \omega^{\mathrm{ph}}(\mathbf{k})=\hbar\left\langle\omega_{\mathbf{n}}^{\mathrm{ph}}\right\rangle-2 A(d)\left(1-C_{2}^{V}\right)^{2}\left(\cos k_{x} d+\cos k_{y} d\right) .
\end{aligned}
$$

Equality (12) defines a quadratic equation for the unknown dispersion dependence $\Omega(\mathbf{k})$. According to (9) polariton frequencies $\Omega(\mathbf{k})$ along with parameters $\left\langle\omega_{\mathbf{n}}^{\mathrm{at}}\right\rangle,\left\langle\omega_{\mathbf{n}}^{\mathrm{ph}}\right\rangle$, $V(\mathbf{k}), A(\mathbf{k})$ and $\left\langle g_{\mathbf{n}}\right\rangle$ are the functions of structural elements in the two subsystems (quantum dots and resonators). In the considered case in view of (9) we obtain

$$
\begin{gathered}
\left\langle\omega_{\mathbf{n}}^{\mathrm{at}}\right\rangle=\left(1-C_{1}^{V}\right) \omega_{\mathrm{at}}, \quad\left\langle\omega_{\mathbf{n}}^{\mathrm{ph}}\right\rangle=\left(1-C_{2}^{V}\right) \omega_{\mathrm{ph}}, \\
\left\langle g_{\mathbf{n}}\right\rangle=g\left(1-C_{1}^{V}\right)\left(1-C_{2}^{V}\right) .
\end{gathered}
$$

$C_{1}^{V}$ and $C_{2}^{V}$ denote concentrations of defects (vacancies) in the atomic and resonator subsystems respectively. The transfer probability of electromagnetic excitation between the nearest neighbor sites is defined by an overlap characteristic of optical fields $A(d) . V(d)$ describes the Coulomb interaction between quantum dots in neighboring cavities.
Substitution of expressions (13) for $\hbar \omega^{\text {at }}(\mathbf{k})$ and $\hbar \omega^{\mathrm{ph}}(\mathbf{k})$ into (12) yields the dispersion law $\Omega_{ \pm}\left(\mathbf{k}, C_{1}^{V}, C_{2}^{V}\right)$ of electromagnetic excitations in the microcavity array (where plus and minus signs stand for the upper and lower dispersion bands, respectively). We have performed the numerical evaluation of $\Omega_{ \pm}\left(\mathbf{k}, C_{1}^{V}, C_{2}^{V}\right)$ for several permissible concentration values falling within the domain $C_{1}^{V} \geq C_{2}^{V}$ (defined by inequality, which accounts for the fact that quantum dots can only reside in the existent cavities). Parameters, which describe an interaction between the atomic and photonic subsystems as well as an overlap of optical fields and an interaction between quantum dots in neighboring cavities were set equal to $g / \hbar=7 \cdot 10^{13} \mathrm{~Hz}, A(d) / 2 \hbar=3.5 \cdot 10^{14} \mathrm{~Hz}$, $V(d) / 2 \hbar=9 \cdot 10^{13} \mathrm{~Hz}$, respectively; the lattice period was taken to be $d=3 \cdot 10^{-7} \mathrm{~m}$. In Fig. 2(a) the dispersion bands $\Omega_{ \pm}\left(k_{x}, k_{y}, C_{1}^{V}, C_{2}^{V}\right)$ are plotted for an ideal structure

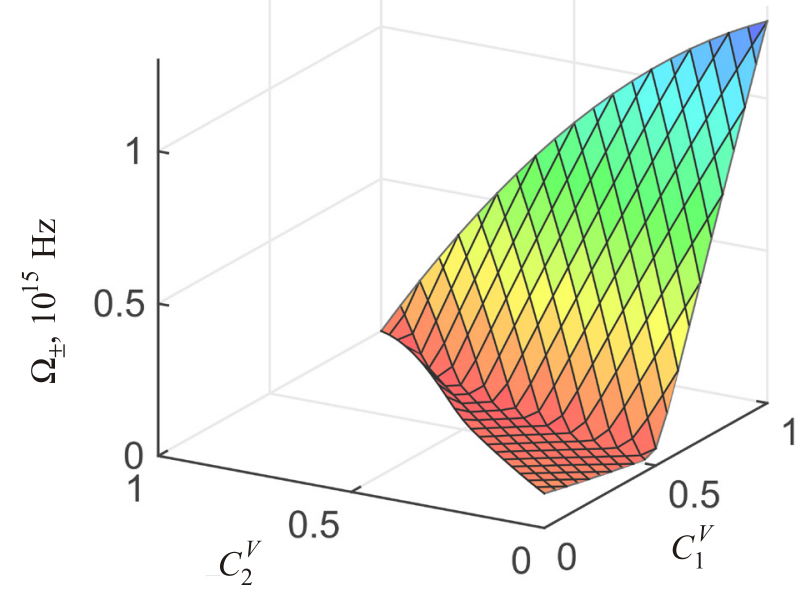

Fig. 3. (Color online) Band gap width plotted as a function of defect concentrations in the domain of definition $C_{1}^{V} \geq C_{2}^{V}$. 
$\left(C_{1}^{V}=C_{2}^{V}=0\right)$, Fig. 2(b) illustrates their transformation under the decrease of parameter $g$ (responsible for interaction between the atomic and photonic subsystems) by a factor of 10. Figures 2(c) gives an example of dispersion bands of a nonideal structure $\left(\left(C_{1}^{V}=0.43, C_{2}^{V}=0.2\right)\right.$.

In Fig. 3 is shown the concentration dependence of the band gap width

$$
\Delta \Omega\left(C_{1}^{V}, C_{2}^{V}\right) \equiv \min _{\mathbf{k}} \Omega_{+}\left(\mathbf{k}, C_{1}^{V}, C_{2}^{V}\right)-\max _{\mathbf{k}} \Omega_{-}\left(\mathbf{k}, C_{1}^{V}, C_{2}^{V}\right) .
$$

It is often important to know how peculiarities of a spectrum are manifested in the corresponding quasiparticle densities of states $\rho_{ \pm}\left(\Omega, C_{1}^{V}, C_{2}^{V}\right)$. In our case the functions $\rho_{ \pm}\left(\Omega, C_{1}^{V}, C_{2}^{V}\right)$ are computed from the formula [23]:

$$
\rho_{ \pm}\left(\Omega, C_{1}^{V}, C_{2}^{V}\right)=\left(\frac{d}{2 \pi}\right)^{2} \oint_{\Omega_{ \pm}(\mathbf{k})=\Omega} \frac{d l}{\left|\nabla_{\mathbf{k}} \Omega_{ \pm}(\mathbf{k})\right|} .
$$

Integration in (14) is carried out along various equifrequency contours falling within the first Brillouin zone $\left(\left(-\pi / d \leq k_{x}, k_{y} \leq \pi / d\right)\right.$. Figs 4(a),(c) show the densities of states of the upper $\rho_{+}(\Omega)$ and lower $\rho_{-}(\Omega)$ dispersion branch polaritons of an ideal structure (whose dispersion bands are plotted in Fig. 2(a)). In Figures 4(b),(d) functions $\rho_{+}(\Omega)$ and $\rho_{-}(\Omega)$ are constructed for a nonideal structure $\left(C_{1}^{V}=0.43, C_{2}^{V}=0.2\right)$, whose dispersion bands are shown in Fig. 2(c). There is a mutual correspondence between individual curve pieces in Figs 4(a)-(d) and the identically colored surface patches in Figs. 2(a),(c). There are several critical frequencies $\Omega_{i}$, which separate differently colored patches in Figs 2(a),(c); in Figs 4(a)-(d) they are marked off by dotted lines. $\Omega_{i}$ 's, at which functions $\rho_{+}(\Omega)$ become infinite are characterized by zero gradient values $\nabla_{\mathbf{k}} \Omega_{ \pm}(\mathbf{k})=0$ at all or some points of the corresponding integration contours. For those of $\Omega_{i}$ 's where functions $\rho_{+}(\Omega)$ have jump discontinuities, certain pieces of the corresponding multiply connected integration contours collapse to points under the simultaneously tending to zero gradient $\nabla_{\mathbf{k}} \Omega_{ \pm}(\mathbf{k}) \rightarrow 0$.

An important property of band gap photonic materials is their ability to produce the so-called "slow" light, which appears to be highly promising for the purposes of construction and utilization of quantum information processing devices [24]. An efficient reduction of quasiparticle group velocity was demonstrated, e.g., in coupled-resonator optical waveguides $[25,26]$ as well as in
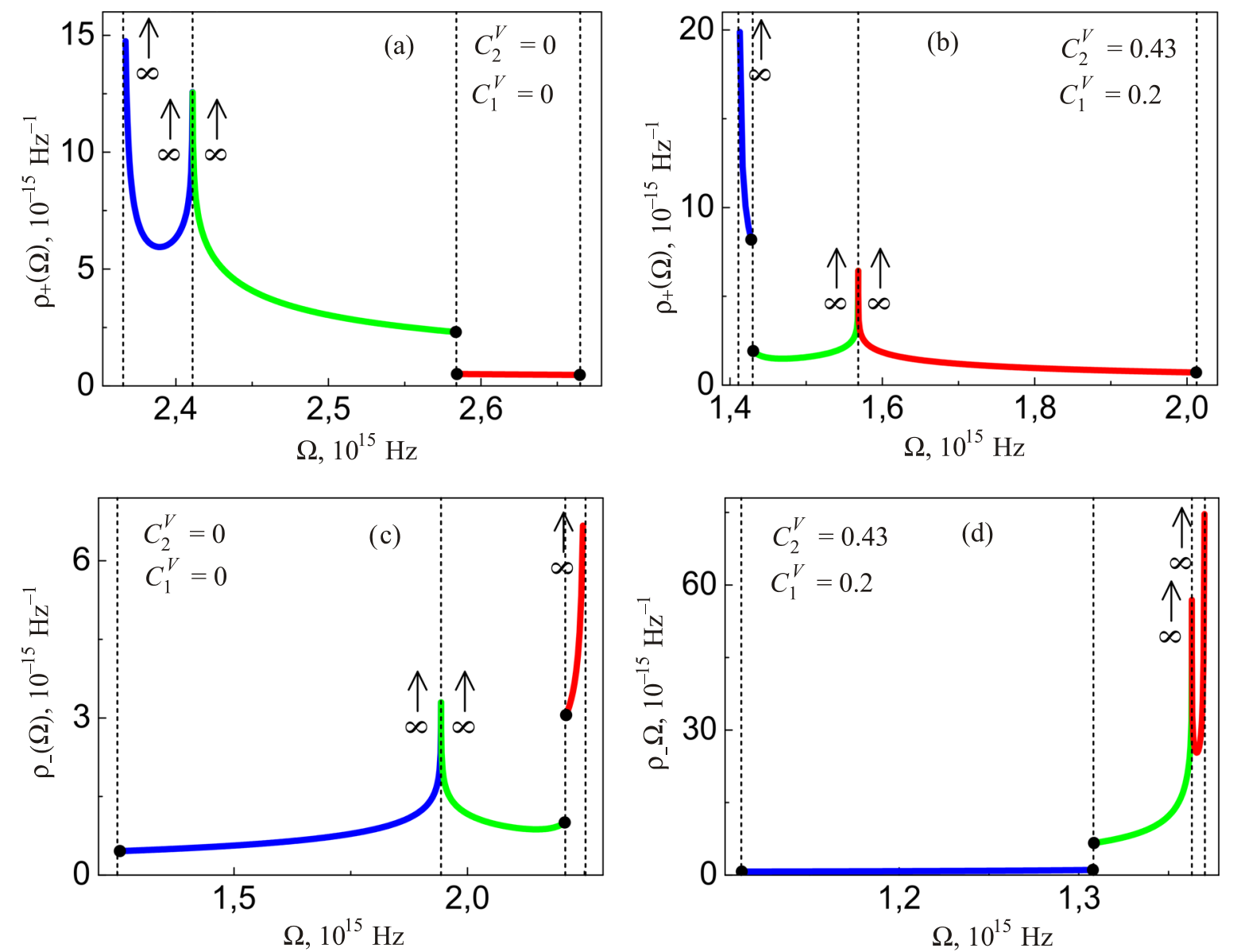

Fig. 4. (Color online) Densities of states of the upper (a) and lower (c) dispersion branch polaritons of an ideal structure (whose dispersion bands are plotted in Fig. 2(a)) and a nonideal structure (b), (d) (whose dispersion bands are shown in Fig. 2(c)). There is a mutual correspondence between individual curve pieces in Figs. 4(a)-(d) and the identically colored surface patches in Figs. 2(a),(c). 


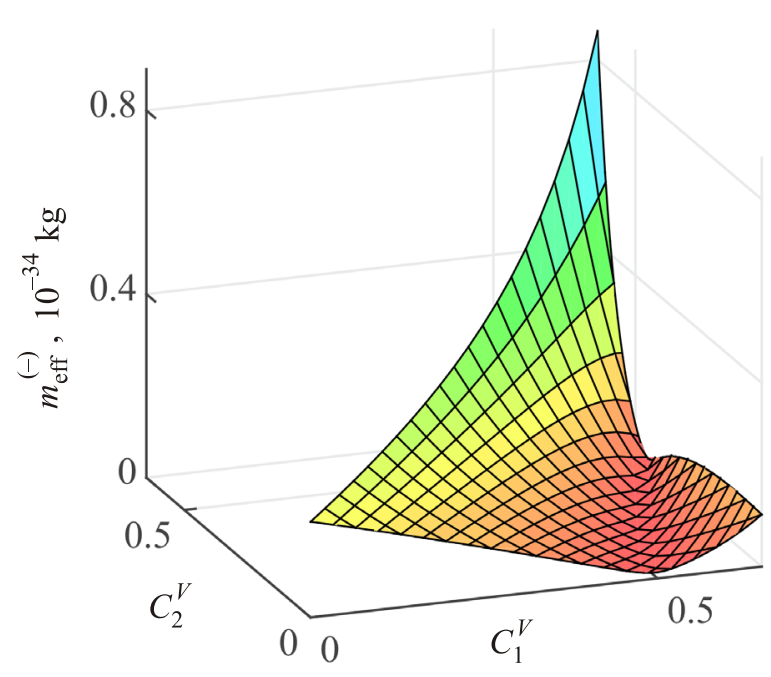

(a)

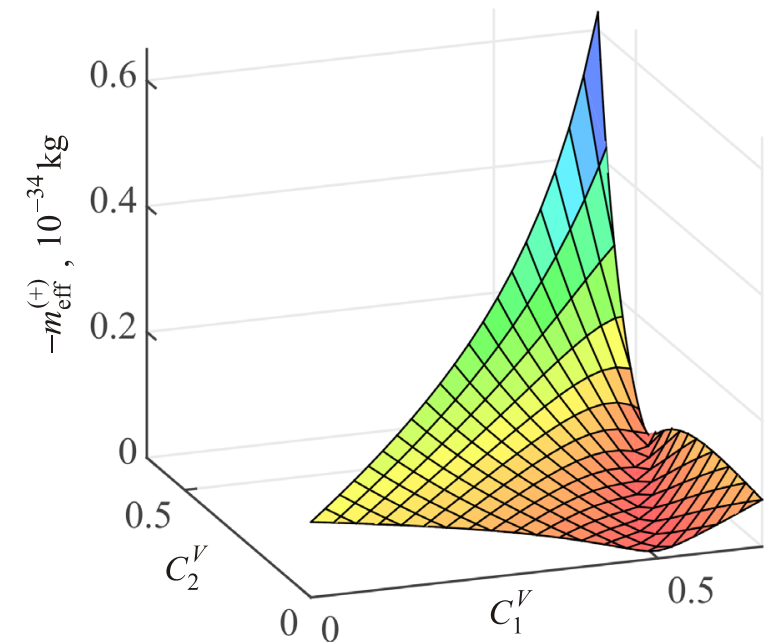

(b)

Fig. 5. (Color online) Concentration dependencies of the effective masses of lower (a) and upper (b) dispersion branch polaritons. The effective mass of the upper dispersion branch polaritons is negative throughout its domain of definition $C_{1}^{V} \geq C_{2}^{V}$; in (b) it is plotted with the opposite sign.

solid-state multilayer semiconductor structures [27]. The key role in the reduction of the said group velocity is played by the character of the effective masses $m_{\mathrm{eff}}^{( \pm)}$of the so-called "dark" and "bright" polaritons, which arise in the specified materials as linear superpositions of the photonic states of resonator subsystems and the coherent excitations of one-level atomic subsystems. Concentration dependencies of the effective masses of upper and lower dispersion branch polaritons are given by the formula

$$
m_{\mathrm{eff}}^{( \pm)}\left(C_{1}^{V}, C_{2}^{V}\right) \equiv \hbar\left(\left.\frac{\partial^{2} \Omega_{ \pm}\left(\mathbf{k}, C_{1}^{V}, C_{2}^{V}\right)}{\partial k_{x}^{2}}\right|_{\substack{k_{x}=0 \\ k_{y}=0}}\right)^{-1}
$$

We have performed their numerical calculation; the results are shown in Figs. 5(a),(b). Examination of Figs. 5(a),(b) permits to conclude that an appropriate choice of defect concentrations $C_{1}^{V}, C_{2}^{V}$ may yield in principle the desirable characteristics of the "slow" light.

\section{Conclusion}

The paper is devoted to elucidation of the effect of pointlike defects on polariton dispersion in a two-dimensional microcavity array with embedded one-level quantum dots. It is shown that the presence of vacancies in the resonator and atomic subsystems results in a substantial renormalization of polariton spectrum and thus in a considerable alteration of optical properties of the structure. Introduction of defects leads to an increase in the effective masses of polaritons and hence to a decrease of their group velocity. Our model is primarily based on the virtual crystal approximation, which is often employed to examine quasiparticle excitations in sufficiently simple disordered superstructures. More complex systems usually require the use of more sophisticated methods such as the (one- or multinode) coherent potential approximation [22], the averaged $T$-matrix method and their various modifications. The obtained numerical results contribute to our understanding of composite polaritonic structures and the prospects of their utilization for construction of solid-state devices with controllable propagation of electromagnetic waves.

\section{Acknowledgments}

The authors respectfully dedicate this paper to the blessed memory of their beloved Teacher, a prominent Ukrainian physicist K.B. Tolpygo, who has made an immense contribution to theoretical foundations of lightmatter coupling. Kirill Borisovich has influenced our scientific formation for which we are endlessly grateful.

This study was supported by European contract FP7PEOPLE-2013-IRSES (Grant \#612600 “LIMACONA”).

1. W. Cai and V. Shalaev, Optical Metamaterials: Fundamentals and Applications, Springer-Verlag, New York (2010).

2. M. Razeghi, Technology of Quantum Devices, Springer, New York, London (2009).

3. A.P. Alodjants, I.O. Barinov, and S.M. Arakelian, J. Phys. B 43, 095502 (2010).

4. E.S. Sedov, A.P. Alodjants, S.M. Arakelian, and Y.Y. Lin, Phys. Rev. A 84, 013813 (2011). 
5. J.D. Joannopoulos, S.G. Johnson, J.N. Winn, and R.D. Meade, Photonic Crystals. Molding the Flow of Light, Princeton University Press, Princeton, New York (2008).

6. K.J. Vahala, Nature 424, 839 (2003).

7. M.A. Kaliteevskii, Tech. Phys. Lett. 23, 120 (1997).

8. V.G. Golubev, A.A. Dukin, A.V. Medvedev, A.B. Pevtsov, A.V. Sel'kin, and N.A. Feoktistov, Semiconductors 37, 832 (2003).

9. J. Vučković, M. Lončar, H. Mabuchi, and A. Scherer, Phys. Rev. E 65, 016608 (2001).

10. D. Englund, A. Majumdar, A. Faraon, M. Toishi, N. Stoltz, P. Petroff, and J. Vučković, Phys. Rev. Lett. 104, 073904 (2010).

11. P. Del'Haye, A. Schliesser, O. Arcizet, T. Wilken, R. Holzwarth, and T.J. Kippenberg, Nature 4501214 (2007).

12. D. Hou, B. Ning, J. Wu, Z. Wang, and J. Zhao, Appl. Phys. Lett. 102, 151104 (2013).

13. S.B. Papp, K. Beha, P. Del'Haye, F. Quinlan, H. Lee, K.J. Vahala, and S.A. Diddams, Optica 1, 10 (2014).

14. J.R. Anglin and A. Vardi, Phys. Rev. A 64, 013605 (2001).

15. V.A. Averchenko, A.P. Alodzhants, S.M. Arakelyan, S.N. Bagayev, E.A. Vinogradov, E.S. Egorov, A.I. Stolyarov, and I.A. Chekhonin, Quantum Electron. 36, 532 (2006).

16. V.V. Rumyantsev, S.A. Fedorov, K.V. Gumennyk, and M.V. Proskurenko, Physica B 442, 57 (2014).
17. V.V. Rumyantsev, S.A. Fedorov, K.V. Gumennyk, M.V. Sychanova, and A.V. Kavokin, Nature Sci. Rep. 4, 6945 (2014).

18. V.V. Rumyantsev, S.A. Fedorov, K.V. Gumennyk, and M.V. Sychanova, Physica B 461, 32 (2015).

19. V.M. Agranovich, Theory of Excitons, Nauka Publishers, Moscow (1968).

20. A.S. Davydov, Theory of Molecular Excitons, McGraw Hill, New York (1962).

21. J.M. Ziman, Models of Disorder: The Theoretical Physics of Homogeneously Disordered Systems, Cambridge University Press, Cambridge (1979).

22. V.F. Los', Theor. Math. Phys. 73, 1076 (1987).

23. A.M. Kosewicz, Mechanika Fizyczna Nieidealnych Krystalicznych Ciai Staiych, Wyd. Uniwersytetu Wroclawskiego, Wroclaw (2000).

24. P.W. Milonni, Fast Light, Slow Light, and Left-Handed Light, Institute of Physics Publishing, Bristol (2005).

25. Z.S. Yang, N.H. Kwong, R. Binder, and A.L. Smirl, J. Opt. Soc. Am. B 22, 2144 (2005).

26. H. Gersen, T.J. Karle, R.J.P. Engelen, W. Bogaerts, J.P. Korterik, N.F. van Hulst, T.F. Krauss, and L. Kuipers, Phys. Rev. Lett. 94, 073903 (2005).

27. A.V. Turukhin, V.S. Sudarshanam, M.S. Shahriar, J.A. Musser, B.S. Ham, and P.R. Hemmer, Phys. Rev. Lett. 88, 023602 (2001). 